\title{
Novel analysis of the fractional Zika model using the Adams type predictor-corrector rule for non-singular and non-local fractional operators
}

\author{
Badr Saad T. Alkahtani, Abdon Atangana*, Ilknur Koca \\ ${ }^{a}$ Department of mathematics, colle of science, King Saud University, P. O. Box 1142, Riyadh, 11989, Saudi Arabia. \\ ${ }^{b}$ Institute for Groundwater Studies, Faculty of Natural and Agricultural Sciences, University of the Free State, 9300, Bloemfontein, South \\ Africa. \\ ${ }^{c}$ Department of Mathematics, Faculty of Sciences, Mehmet Akif Ersoy University, 15100, Burdur, Turkey. \\ Communicated by D. Baleanu
}

\begin{abstract}
A mathematical system of equations using the concept of fractional differentiation with non-local and non-singular kernel has been analysed in this work. The developed mathematical model is designed to portray the spread of Zika virus within a given population. We presented the equilibrium point and also the reproductive number. The model was solving analytically using the Adams type predictor-corrector rule for Atangana-Baleanu fractional integral. The existence and uniqueness exact solution was presented under some conditions. The numerical replications were also presented. (c) 2017 All rights reserved.
\end{abstract}

Keywords: Zika virus, reproduction number, numerical approximation.

2010 MSC: 34A34, 34A08, 74G15.

\section{Introduction}

Recently the world has faced a great challenge with the spread of Zika virus within the continent of South America precisely in Brazil. It is believed that, the Zika virus Zika forest in Uganda, this virus belongs to the family of Flaviviridea and spread by daytime via a vector known as Aedes mosquitoes $[7,12,13]$. One most note that this virus is connected to the fatal one known as dengue, yellow fever, Japanese encephalitis and West Nile virus. The virus is known to be transmitted by the female Aades aegypti mosquito. We must mention that this vector is active only in daytime. Some studies reveal that, the virus could also be transmitted via sexual intercourse or via blood transfusions. In the work done in [10] revealed that, the reproductive number of Zika virus is between 1.6 and 6.6. Fewer researchers in the literature have done some researches related to mathematical modelling see for instance [6]. However the study done recently have used the approach of local differentiation without any justification. It is important to note that the local differentiation has failed to portray real world problems due to the lack of

\footnotetext{
${ }^{*}$ Corresponding author

Email addresses: balqahtani1@ksu.edu.sa (Badr Saad T. Alkahtani), abdonatangana@yahoo.fr (Abdon Atangana), ikoca@mehmetakif . edu . tr (Ilknur Koca)
}

doi:10.22436/jnsa.010.06.32 
non-locality effect into mathematical formulation. In the real world explanation, the local differentiation does explain the rate of change between two points not necessary the same, in this case one will ask the question to know what is happening between the two point. The failure of this answer by the concept of local differentiation leads some eminent researchers like Riemann and Liouville to introduce the concept of differentiation with non-local operator $[2,5,8,9,11]$. The concept of differentiation with non-local operator is also known as fractional differentiation has been recognized a very powerful mathematical instrument able to give meaning and answer the question "what happen between the two points" as they are able to describe or include into the mathematical formulation the history of the moving object of the spread of a given virus. In this paper therefore we will use the concept of fractional differentiation to analyse the spread of Zika virus.

\section{Atangana-Baleanu derivatives in Caputo sense}

In this section, we present the definitions of the new fractional derivative with no singular and nonlocal kernel $[1,3,4]$.

Definition 2.1. Let $f \in H^{1}(a, b), b>a, \alpha \in[0,1]$ then, the definition of the new fractional derivative (Atangana-Baleanu derivative in Caputo sense) is given as:

$$
\underset{a}{A B C} D_{t}^{\alpha}(f(t))=\frac{B(\alpha)}{1-\alpha} \int_{a}^{t} f^{\prime}(x) E_{\alpha}\left[-\alpha \frac{(t-x)^{\alpha}}{1-\alpha}\right] d x .
$$

Definition 2.2. Let $f \in H^{1}(a, b), b>a, \alpha \in[0,1]$ and not necessary differentiable, then the definition of the new fractional derivative (Atangana-Baleanu fractional derivative in Riemann-Liouville sense) is given as:

$$
{ }_{a}^{A B R} D_{t}^{\alpha}(f(t))=\frac{B(\alpha)}{1-\alpha} \frac{d}{d t} \int_{a}^{t} f(x) E_{\alpha}\left[-\alpha \frac{(t-x)^{\alpha}}{1-\alpha}\right] d x .
$$

Definition 2.3. The fractional integral associate to the new fractional derivative with non-local kernel is defined as:

$$
{ }_{a}^{A B} I_{t}^{\alpha}\{f(t)\}=\frac{1-\alpha}{B(\alpha)} f(t)+\frac{\alpha}{B(\alpha) \Gamma(\alpha)} \int_{a}^{t} f(y)(t-y)^{\alpha-1} d y .
$$

The initial function is recovered when the fractional order turns to zero. Also when the order turns to 1 we have the classical integral.

\section{Equilibrium points of system}

In this section, the model based of Zika based on the classical differentiation will be extended to the scope of fractional differentiation with non-local and non-singular kernel. The model with fractional differentiation is including the effect of memory and also the heterogeneity of the dynamical system. The new model is given below. In particular we will provide the analysis of equilibrium points. This will be done using the properties of Caputo fractional type of definition.

In this paper, Zika model has been analyzed. Model is given with system as below:

$$
\begin{aligned}
& { }_{0}^{A B C} D_{t}^{\alpha}\left[{ }^{H} S(t)\right]=\Lambda_{H}-\left(1-\mu_{1}\right) \beta_{H}{ }^{H} S\left({ }^{\mathrm{V}} \mathrm{I}+\sigma{ }^{H} \mathrm{I}\right)-\mu_{H}{ }^{{ }^{H}} S \text {, } \\
& { }_{0}^{A B C} D_{t}^{\alpha}\left[{ }^{H} \mathrm{I}(\mathrm{t})\right]=\left(1-\mu_{1}\right) \beta_{\mathrm{H}}{ }^{\mathrm{H}} \mathrm{S}\left({ }^{\mathrm{V}} \mathrm{I}+\sigma^{\mathrm{H}} \mathrm{I}\right)-\left(\mu_{\mathrm{H}}+\gamma+\eta_{\mathrm{H}} \mu_{2}\right){ }^{\mathrm{H}} \mathrm{I} \text {, } \\
& { }_{0}^{A B C} D_{t}^{\alpha}\left[{ }^{H} R(t)\right]=\left(\gamma+\eta_{H} \mu_{2}\right){ }^{H} I-\mu_{H}{ }^{H} R \text {, } \\
& { }_{0}^{A B C} D_{t}^{\alpha}\left[{ }^{V} S(t)\right]=\Lambda_{V}-\left(1-\mu_{1}\right) \beta_{V}{ }^{V_{S}}{ }^{H} I-\left(\mu_{V}+\eta_{V} \mu_{3}\right){ }^{\vee} S \text {, } \\
& { }_{0}^{A B C} D_{t}^{\alpha}\left[{ }^{V} \mathrm{I}(t)\right]=\left(1-\mu_{1}\right) \beta_{V}{ }^{V} S^{H^{H}}-\left(\mu_{V}+\eta_{V} \mu_{3}\right){ }^{V_{I}} \text {. }
\end{aligned}
$$


At the model, while human population at time $t$ is stated by ${ }^{\mathrm{H}} \mathrm{N}(\mathrm{t})={ }^{\mathrm{H}} \mathrm{S}(\mathrm{t})+{ }^{\mathrm{H}} \mathrm{I}(\mathrm{t})+{ }^{\mathrm{H}} \mathrm{R}(\mathrm{t})$, mosquito population at time $t$ is stated by ${ }^{\mathrm{H}} \mathrm{N}(\mathrm{t})$. Mosquito population is sub-classified with susceptible mosquitoes ${ }^{\mathrm{V}} \mathrm{S}(\mathrm{t})$ and infectious mosquitoes ${ }^{\mathrm{V}} \mathrm{I}(\mathrm{t})$. ${ }^{\mathrm{H}} \mathrm{S}(\mathrm{t})$ is individual with Zika symptoms and infectious ${ }^{\mathrm{H}} \mathrm{I}(\mathrm{t})$.

$\mathrm{H}_{\mathrm{R}}(\mathrm{t})$ is individual recovered from Zika. Also $\beta_{H}$ is the transmission rate from humans to mosquitoes, $\beta_{V}$ is the transmission rate from ZIKV from the mosquitoes to humans. Natural death parameter is given by $\mu_{H}$, the recruitment rate in susceptible population is given by $\Lambda_{H}$, natural death parameter of mosquitoes is given by $\mu_{V}$. Finally $\Lambda_{V}$ is the recruitment rate in susceptible mosquito population and $\eta_{V}$ is the mosquito death rate from insecticide. In model, $\mu_{1}$ is prevention control, $\mu_{2}$ is treatment control and $\mu_{3}$ is insecticide control that are bounded with Lebesque integrable. Detailed information about Zika model can be found in [6].

Now we will consider the equilibrium points of system with Atangana-Baleanu derivative in Caputo system. The equilibrium points are obtained by equating the right-hand sides of (3.1) to zero as below:

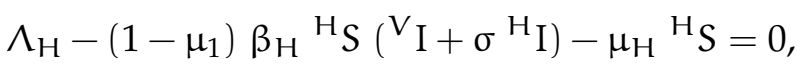

$$
\begin{aligned}
& \left(1-\mu_{1}\right) \beta_{\mathrm{H}}{ }^{\mathrm{H}_{\mathrm{S}}}\left({ }^{\mathrm{V}} \mathrm{I}+\sigma^{\mathrm{H}_{\mathrm{I}}}\right)-\left(\mu_{\mathrm{H}}+\gamma+\eta_{\mathrm{H}} \mu_{2}\right){ }^{\mathrm{H}_{\mathrm{I}}}=0 \text {, } \\
& \left(\gamma+\eta_{\mathrm{H}} \mu_{2}\right){ }^{\mathrm{H}} \mathrm{I}-\mu_{\mathrm{H}}{ }^{\mathrm{H}} \mathrm{R}=0 \text {, } \\
& \Lambda_{V}-\left(1-\mu_{1}\right) \beta_{V}{ }^{{ }}{ }_{S}{ }^{H_{I}}-\left(\mu_{V}+\eta_{V} \mu_{3}\right)^{{ }} \mathrm{S}=0 \text {, } \\
& \left(1-\mu_{1}\right) \beta_{V}{ }^{\vee}{ }_{S}{ }^{H} \mathrm{I}-\left(\mu_{V}+\eta_{V} \mu_{3}\right){ }^{\vee} \mathrm{I}=0 \text {. }
\end{aligned}
$$

Zika system has $\mathrm{Z}_{0}=\left(\frac{\Lambda_{\mathrm{H}}}{\mu_{\mathrm{H}}}, 0,0, \frac{\Lambda_{V}}{\mu_{\mathrm{V}}}\right)$ and endemic equilibrium point is $\mathrm{Z}_{1}=\left({ }^{\mathrm{H}} \mathrm{S}^{*}, \mathrm{H} \mathrm{I}^{*}, \mathrm{H} \mathrm{R}^{*}, \mathrm{~V} \mathrm{~S}^{*}, \mathrm{~V} \mathrm{I}^{*}\right)$ where

$$
\begin{aligned}
\mathrm{H}_{S^{*}} & =\frac{-\gamma \beta_{V} \Lambda_{V}-\beta_{V} \Lambda_{V} \mu_{H}+\beta_{V} \Lambda_{H} \mu_{V}+\gamma \mu_{V}^{2}+\mu_{H} \mu_{V}^{2}}{\beta_{V} \mu_{H} \mu_{V}}, \\
{ }^{H^{*}} & =\frac{\beta_{V} \Lambda_{V}-\mu_{V}^{2}}{\beta_{V} \mu_{V}}, \\
{ }^{H^{*}} & =\frac{\gamma\left(\beta_{V} \Lambda_{V}-\mu_{V}^{2}\right)}{\beta_{V} \mu_{V} \mu_{H}}, \\
{ }^{V_{S}} & =\frac{\mu_{V}}{\beta_{V}}, \\
\mathrm{~V}_{I^{*}} & =\frac{\mu_{H}\left(\gamma+\mu_{H}\right)\left(\beta_{V} \Lambda_{V}-\mu_{V}^{2}\right)}{\beta_{H}\left(\gamma \beta_{V} \Lambda_{V}+\beta_{V} \Lambda_{V} \mu_{H}-\beta_{V} \Lambda_{H} \mu_{V}-\gamma \mu_{V}^{2}-\mu_{H}^{3}\right)} .
\end{aligned}
$$

\subsection{The reproduction number of model}

To find the reproduction number of model, Okosun and Makinde found F and $V$ by using the nextgeneration operator approach $[12,13]$. Here

$$
F=\left[\begin{array}{cc}
\frac{\sigma \beta_{H} \wedge_{H}}{\mu_{H}} & \frac{\beta_{H} \wedge_{H}}{\mu_{H}} \\
\frac{\beta V \Lambda_{V}}{\mu_{V}} & 0
\end{array}\right],
$$

and

$$
\mathrm{V}=\left[\begin{array}{cc}
\gamma+\mu_{\mathrm{H}} & 0 \\
0 & \mu_{\mathrm{V}}
\end{array}\right]
$$

So the reproduction number of model is given as below:

$$
R_{0}=\rho\left(\mathrm{FV}^{-1}\right)=\frac{\sqrt{\beta_{\mathrm{H}} \Lambda_{\mathrm{H}}\left(\begin{array}{c}
4 \gamma \mu_{\mathrm{H}} \beta_{V} \Lambda_{V} \\
+\sigma^{2} \beta_{H} \Lambda_{H} \mu_{V}^{2}+4 \mu_{H}^{2} \beta_{V} \Lambda_{V}
\end{array}\right)+\sigma^{2} \beta_{H} \Lambda_{H} \mu_{V}}}{2 \mu_{H} \mu_{V}\left(\gamma+\mu_{H}\right)} .
$$




\section{Numerical approximation}

In this section, we present a numerical solution of the modified model. To achieve this, the system is converted to the fractional Volterra type. The Adams type predictor-corrector rule for Atangana-Baleanu fractional integral operator is therefore used to develop the numerical iterative formula.

Let we consider Zika model with new derivative to obtain more efficient result. Advantage of derivative is that has non-local and non-singular kernel.

$$
\begin{aligned}
& { }_{0}^{A B C} D_{t}^{\alpha}\left[{ }^{H} S(t)\right]=\Lambda_{H}-\left(1-\mu_{1}\right) \beta_{H}{ }^{H} S\left({ }^{\mathrm{V}} \mathrm{I}+\sigma{ }^{H} \mathrm{I}\right)-\mu_{H}{ }^{{ }^{H}} S, \\
& { }_{0}^{A B C} D_{t}^{\alpha}\left[{ }^{H} \mathrm{I}(\mathrm{t})\right]=\left(1-\mu_{1}\right) \beta_{\mathrm{H}}{ }^{\mathrm{H}} \mathrm{S}\left({ }^{\mathrm{V}} \mathrm{I}+\sigma^{\mathrm{H}} \mathrm{I}\right)-\left(\mu_{\mathrm{H}}+\gamma+\eta_{\mathrm{H}} \mu_{2}\right){ }^{\mathrm{H}} \mathrm{I} \text {, } \\
& { }_{0}^{A B C} D_{t}^{\alpha}\left[{ }^{H} R(t)\right]=\left(\gamma+\eta_{H} \mu_{2}\right){ }^{H} \mathrm{I}-\mu_{H}{ }^{H}{ }_{R} \text {, } \\
& { }_{0}^{A B C} D_{t}^{\alpha}\left[{ }^{V} S(t)\right]=\Lambda_{V}-\left(1-\mu_{1}\right) \beta_{V}{ }^{V_{S}}{ }^{H} I-\left(\mu_{V}+\eta_{V} \mu_{3}\right){ }^{V_{S}} \text {, } \\
& { }_{0}^{A B C} D_{t}^{\alpha}\left[{ }^{\left.V_{I}(t)\right]}=\left(1-\mu_{1}\right) \beta_{V}{ }^{V_{S}}{ }^{H} \mathrm{I}-\left(\mu_{V}+\eta_{V} \mu_{3}\right){ }^{V_{I}}\right.
\end{aligned}
$$

For convenience, we will take model as below:

$$
\begin{aligned}
& { }_{0}^{A B C} D_{t}^{\alpha}\left[{ }^{H} S(t)\right]=F_{1}\left({ }^{H} S,{ }^{H} I_{,}{ }^{H} R,{ }^{V} S,{ }^{V} I, t\right), \\
& { }_{0}^{A B C} D_{t}^{\alpha}\left[{ }^{H} \mathrm{I}(\mathrm{t})\right]=\mathrm{F}_{2}\left({ }^{\mathrm{H}} \mathrm{S},{ }^{\mathrm{H}} \mathrm{I},{ }^{\mathrm{H}} \mathrm{R},{ }^{\mathrm{V}} \mathrm{S},{ }^{\mathrm{V}} \mathrm{I}, \mathrm{t}\right) \text {, } \\
& { }_{0}^{A B C} D_{t}^{\alpha}\left[{ }^{H} R(t)\right]=F_{3}\left({ }^{H} S,{ }^{H} I,{ }^{H} R,{ }^{V} S,{ }^{V} I, t\right) \text {, } \\
& { }_{0}^{A B C} D_{t}^{\alpha}\left[{ }^{V} S(t)\right]=F_{4}\left({ }^{H} S,{ }^{H} I_{,}{ }^{H} R,{ }^{V} S,{ }^{V} I, t\right) \text {, } \\
& { }_{0}^{A B C} D_{t}^{\alpha}\left[{ }^{V} I(t)\right]=F_{5}\left({ }^{H} S,{ }^{H} I_{,}{ }^{H} R,{ }^{V} S,{ }^{V} I, t\right) .
\end{aligned}
$$

Now we will consider system of Zika model with Atangana-Balenau integral as below.

$$
\begin{aligned}
& { }^{\mathrm{H}} \mathrm{S}(\mathrm{t})={ }^{\mathrm{H}} \mathrm{S}_{0}(\mathrm{t})+\frac{1-\alpha}{\mathrm{B}(\alpha)} \mathrm{F}_{1}\left({ }^{\mathrm{H}} \mathrm{S},{ }^{\mathrm{H}} \mathrm{I},{ }^{\mathrm{H}} \mathrm{R},{ }^{\mathrm{V}} \mathrm{S},{ }^{\mathrm{V}} \mathrm{I}, \mathrm{t}\right) \\
& +\frac{\alpha}{B(\alpha) \Gamma(\alpha)} \int_{0}^{t} F_{1}\left({ }^{H} S,{ }^{H} I^{H}{ }^{H},{ }^{V} S,{ }^{V} I, \tau\right)(t-\tau)^{\alpha-1} d \tau, \\
& { }^{H} \mathrm{I}(\mathrm{t})={ }^{\mathrm{H}} \mathrm{I}_{0}(\mathrm{t})+\frac{1-\alpha}{\mathrm{B}(\alpha)} \mathrm{F}_{2}\left({ }^{\mathrm{H}} \mathrm{S}_{,}{ }^{\mathrm{H}} \mathrm{I},{ }^{\mathrm{H}} \mathrm{R},{ }^{\mathrm{V}} \mathrm{S},{ }^{\mathrm{V}} \mathrm{I}, \mathrm{t}\right) \\
& +\frac{\alpha}{\mathrm{B}(\alpha) \Gamma(\alpha)} \int_{0}^{\mathrm{t}} \mathrm{F}_{2}\left({ }^{\mathrm{H}} \mathrm{S},{ }^{\mathrm{H}} \mathrm{I},{ }^{\mathrm{H}} \mathrm{R}^{\mathrm{V}}{ }^{\mathrm{V}} \mathrm{S},{ }^{\mathrm{V}} \mathrm{I}, \tau\right)(\mathrm{t}-\tau)^{\alpha-1} \mathrm{~d} \tau, \\
& { }^{H} \mathrm{R}(\mathrm{t})={ }^{\mathrm{H}} \mathrm{R}_{0}(\mathrm{t})+\frac{1-\alpha}{\mathrm{B}(\alpha)} \mathrm{F}_{3}\left({ }^{\mathrm{H}} \mathrm{S},{ }^{\mathrm{H}} \mathrm{I},{ }^{\mathrm{H}} \mathrm{R},{ }^{\mathrm{V}} \mathrm{S},{ }^{\mathrm{V}} \mathrm{I}, \mathrm{t}\right) \\
& +\frac{\alpha}{B(\alpha) \Gamma(\alpha)} \int_{0}^{\mathrm{t}} \mathrm{F}_{3}\left({ }^{\mathrm{H}} \mathrm{S},{ }^{\mathrm{H}} \mathrm{I},{ }^{\mathrm{H}} \mathrm{R},{ }^{\mathrm{V}} \mathrm{S},{ }^{\mathrm{V}} \mathrm{I}, \tau\right)(\mathrm{t}-\tau)^{\alpha-1} \mathrm{~d} \tau, \\
& { }^{V_{S}} \mathrm{t}(\mathrm{t})={ }^{\mathrm{V}} \mathrm{S}_{0}(\mathrm{t})+\frac{1-\alpha}{\mathrm{B}(\alpha)} \mathrm{F}_{4}\left({ }^{\mathrm{H}} \mathrm{S},{ }^{\mathrm{H}} \mathrm{I},{ }^{\mathrm{H}} \mathrm{R},{ }^{\mathrm{V}} \mathrm{S},{ }^{\mathrm{V}} \mathrm{I}, \mathrm{t}\right) \\
& +\frac{\alpha}{\mathrm{B}(\alpha) \Gamma(\alpha)} \int_{0}^{\mathrm{t}} \mathrm{F}_{4}\left({ }^{\mathrm{H}} \mathrm{S},{ }^{\mathrm{H}} \mathrm{I},{ }^{\mathrm{H}} \mathrm{R},{ }^{\mathrm{V}} \mathrm{S},{ }^{\mathrm{V}} \mathrm{I}, \tau\right)(\mathrm{t}-\tau)^{\alpha-1} \mathrm{~d} \tau,
\end{aligned}
$$

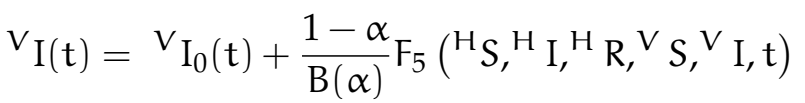

$$
\begin{aligned}
& +\frac{\alpha}{B(\alpha) \Gamma(\alpha)} \int_{0}^{t} F_{5}\left({ }^{H} S,{ }^{H}{ }^{H},{ }^{H},{ }^{V},{ }^{V}, I, \tau\right)(t-\tau)^{\alpha-1} d \tau \text {. }
\end{aligned}
$$


Now we need numerical approximation scheme for Atangana-Baleanu fractional integral. For this we use Adams type predictor-corrector rule for Atangana-Baleanu fractional integral. So we have

$$
\begin{aligned}
{ }_{b}^{A B} I_{t}^{\alpha}[f(t)] & =\frac{1-\alpha}{B(\alpha)} f(t)+\frac{\alpha}{B(\alpha) \Gamma(\alpha)} \int_{b}^{t} f(j)(t-j)^{\alpha-1} d j, \\
h & =\frac{T}{N^{\prime}}, \quad t_{l}=h l \quad(l=0,1,2,3, \cdots, N),
\end{aligned}
$$

where $\mathrm{T}$ is upper bound of the interval. Then the corrector formula of integral version of derivative is given as below

$$
\begin{aligned}
f_{h}\left(t_{n+1}\right)= & f_{0}\left(t_{n+1}\right)+\frac{(1-\alpha) h^{\alpha}}{B(\alpha) \Gamma(\alpha+2)} F\left(t_{n+1}, f_{h}^{P}\left(t_{n+1}\right)\right) \\
& +\frac{\alpha h^{\alpha}}{B(\alpha) \Gamma(\alpha+2)} \sum_{j=0}^{n} \theta_{j, n+1} F\left(t_{j}, f_{h}\left(t_{j}\right)\right)
\end{aligned}
$$

Here

$$
\theta_{j, n+1}= \begin{cases}n^{\alpha+1}-(n-\alpha)(n+1)^{\alpha}, & \text { if } j=0, \\ (n-j+2)^{\alpha+1}+(n-j)^{\alpha+1}-2(n-j+1)^{\alpha+1}, & 1 \leqslant j \leqslant n, \\ 1, & j=n+1 .\end{cases}
$$

Also the predictor $f_{h}^{P}\left(t_{n+1}\right)$ is given as follows:

$$
\begin{aligned}
f_{h}^{P}\left(t_{n+1}\right)= & f_{0}+\frac{1-\alpha}{B(\alpha)} F\left(t_{n}, f_{h}\left(t_{n}\right)\right) \\
& +\frac{\alpha}{B(\alpha) \Gamma^{2}(\alpha)} \sum_{j=0}^{n} \delta_{j, n+1} F\left(t_{j}, f_{h}\left(t_{j}\right)\right), \\
\delta_{j, n+1}= & \frac{h^{\alpha}}{\alpha}\left((n+1-j)^{\alpha}-(n-j)^{\alpha}\right), \quad 0 \leqslant j \leqslant n .
\end{aligned}
$$

Using the above formula, we reformulated the system of (3.1) to the following iterative formula:

$$
\begin{aligned}
& { }^{\mathrm{H}} \mathrm{S}_{\mathrm{h}}\left(\mathrm{t}_{\mathrm{n}+1}\right)={ }^{\mathrm{H}} \mathrm{S}_{0}\left(\mathrm{t}_{\mathrm{n}+1}\right) \\
& +\frac{(1-\alpha) h^{\alpha}}{B(\alpha) \Gamma(\alpha+2)} F_{1}\left({ }^{H} S_{n+1}^{p},{ }^{H} I_{n+1}^{p},{ }^{H} R_{n+1}^{p},{ }^{p} S_{n+1}^{p},{ }^{p} I_{n+1}^{p}, t_{n+1}\right) \\
& +\frac{h^{\alpha}}{\Gamma(\alpha+2)} \frac{\alpha}{B(\alpha)} \sum_{j=0}^{n} a_{1, j, n+1} F_{1}\left({ }^{H} S_{j},{ }^{H} I_{j},{ }^{H} R_{j},{ }^{V} S_{j},{ }^{V} I_{j}, t_{j}\right) \text {, } \\
& { }^{\mathrm{H}} \mathrm{I}_{\mathrm{h}}\left(\mathrm{t}_{\mathrm{n}+1}\right)={ }^{\mathrm{H}} \mathrm{I}_{0}\left(\mathrm{t}_{\mathrm{n}+1}\right) \\
& +\frac{(1-\alpha) h^{\alpha}}{B(\alpha) \Gamma(\alpha+2)} F_{2}\left({ }^{H} S_{n+1}^{p},{ }^{H} I_{n+1}^{p},{ }^{\prime} R_{n+1}^{p},{ }^{p} S_{n+1}^{p},{ }^{p} I_{n+1}^{p}, t_{n+1}\right) \\
& +\frac{h^{\alpha}}{\Gamma(\alpha+2)} \frac{\alpha}{B(\alpha)} \sum_{j=0}^{n} a_{2, j, n+1} F_{2}\left({ }^{H} S_{j},{ }^{H} I_{j},{ }^{H} R_{j},{ }^{V} S_{j},{ }^{V} I_{j}, t_{j}\right) \text {, } \\
& { }^{H} R_{h}\left(t_{n+1}\right)={ }^{H} R_{0}\left(t_{n+1}\right)+\frac{(1-\alpha) h^{\alpha}}{B(\alpha) \Gamma(\alpha+2)} F_{3}\left({ }^{H} S_{n+1}^{p},{ }^{H} I_{n+1}^{p},{ }^{H} R_{n+1}^{p},{ }^{p} S_{n+1}^{p},{ }^{\prime} I_{n+1}^{p}, t_{n+1}\right) \\
& +\frac{h^{\alpha}}{\Gamma(\alpha+2)} \frac{\alpha}{B(\alpha)} \sum_{j=0}^{n} a_{3, j, n+1} F_{3}\left({ }^{H} S_{j},{ }^{H} I_{j},{ }^{H} R_{j},{ }^{V} S_{j},{ }^{V} I_{j}, t_{j}\right), \\
& \left.\mathrm{v}^{\mathrm{h}} \mathrm{t}_{\mathrm{n}+1}\right)={ }^{\mathrm{v}} \mathrm{S}_{0}\left(\mathrm{t}_{\mathrm{n}+1}\right)
\end{aligned}
$$




$$
\begin{aligned}
& +\frac{(1-\alpha) h^{\alpha}}{B(\alpha) \Gamma(\alpha+2)} F_{4}\left({ }^{H} S_{n+1}^{p},{ }^{H} I_{n+1}^{p}, H R_{n+1}^{p},{ }^{p} S_{n+1}^{p},{ }^{p} I_{n+1}^{p}, t_{n+1}\right) \\
& +\frac{h^{\alpha}}{\Gamma(\alpha+2)} \frac{\alpha}{B(\alpha)} \sum_{j=0}^{n} a_{4, j, n+1} F_{4}\left({ }^{H} S_{j},{ }^{H} I_{j},{ }^{H} R_{j},{ }^{V} S_{j},{ }^{V} I_{j}, t_{j}\right),
\end{aligned}
$$

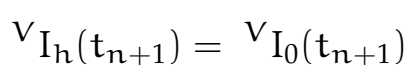

$$
\begin{aligned}
& +\frac{(1-\alpha) h^{\alpha}}{B(\alpha) \Gamma(\alpha+2)} F_{5}\left({ }^{H} S_{n+1}^{p}, H I_{n+1}^{p}, H R_{n+1}^{p}, S_{n+1}^{p}, I_{n+1}^{p}, t_{n+1}\right) \\
& +\frac{h^{\alpha}}{\Gamma(\alpha+2)} \frac{\alpha}{B(\alpha)} \sum_{j=0}^{n} a_{5, j, n+1} F_{5}\left({ }^{H} S_{j},{ }^{H} I_{j},{ }^{H} R_{j},{ }^{V} S_{j},{ }^{V} I_{j}, t_{j}\right) \text {. }
\end{aligned}
$$

Also predictor terms ${ }^{H} S_{n+1}^{p},{ }^{H} I_{n+1}^{p},{ }^{H} R_{n+1}^{p},{ }^{v} S_{n+1}^{p},{ }^{v} I_{n+1}^{p}$ are given below:

$$
\begin{aligned}
& { }^{H} S_{n+1}^{p}=S_{0}(t)+\frac{1-\alpha}{B(\alpha)} F_{1}\left({ }^{H} S_{n},{ }^{H} I_{n},{ }^{H} R_{n},{ }^{V} S_{n},{ }^{V} I_{n}, t_{n}\right) \\
& +\frac{\alpha}{\Gamma^{2}(\alpha) B(\alpha)} \sum_{j=0}^{n} b_{1, j, n+1} F_{1}\left({ }^{H} S_{j},{ }^{H} I_{j},{ }^{H} R_{j},{ }^{V} S_{j},{ }^{V} I_{j}, t_{j}\right) \text {, } \\
& { }^{H} I_{n+1}^{p}=I_{0}(t)+\frac{1-\alpha}{B(\alpha)} F_{2}\left({ }^{H} S_{n},{ }^{H} I_{n},{ }^{H} R_{n},{ }^{V} S_{n},{ }^{V} I_{n}, t_{n}\right) \\
& +\frac{\alpha}{\Gamma^{2}(\alpha) B(\alpha)} \sum_{j=0}^{n} b_{2, j, n+1} F_{2}\left({ }^{H} S_{j},{ }^{H} I_{j},{ }^{H} R_{j},{ }^{V} S_{j},{ }^{V} I_{j}, t_{j}\right) \text {, } \\
& { }^{H} R_{n+1}^{p}=R_{0}(t)+\frac{1-\alpha}{B(\alpha)} F_{3}\left({ }^{H} S_{n},{ }^{H} I_{n},{ }^{H} R_{n},{ }^{V} S_{n},{ }^{V} I_{n}, t_{n}\right) \\
& +\frac{\alpha}{\Gamma^{2}(\alpha) B(\alpha)} \sum_{j=0}^{n} b_{3, j, n+1} F_{3}\left({ }^{H} S_{j},{ }^{H} I_{j},{ }^{H} R_{j},{ }^{V} S_{j},{ }^{V} I_{j}, t_{j}\right), \\
& v_{S_{n+1}^{p}}^{p}=S_{0}(t)+\frac{1-\alpha}{B(\alpha)} F_{4}\left({ }^{H} S_{n},{ }^{H} I_{n},{ }^{H} R_{n},{ }^{V} S_{n},{ }^{V} I_{n}, t_{n}\right) \\
& +\frac{\alpha}{\Gamma^{2}(\alpha) B(\alpha)} \sum_{j=0}^{n} b_{4, j, n+1} F_{4}\left({ }^{H} S_{j},{ }^{H} I_{j},{ }^{H} R_{j},{ }^{V} S_{j},{ }^{V} I_{j}, t_{j}\right), \\
& \mathrm{v}_{\mathrm{I}_{\mathrm{n}+1}^{\mathrm{p}}}=\mathrm{I}_{0}(\mathrm{t})+\frac{1-\alpha}{\mathrm{B}(\alpha)} \mathrm{F}_{5}\left({ }^{\mathrm{H}} \mathrm{S}_{\mathrm{n}},{ }^{\mathrm{H}} \mathrm{I}_{\mathrm{n}},{ }^{H} \mathrm{R}_{\mathrm{n}}, \mathrm{V}_{\mathrm{S}_{\mathrm{n}},}, \mathrm{V}_{\mathrm{n}}, \mathrm{t}_{\mathrm{n}}\right) \\
& +\frac{\alpha}{\Gamma^{2}(\alpha) B(\alpha)} \sum_{j=0}^{n} b_{5, j, n+1} F_{5}\left({ }^{H} S_{j},{ }^{H} I_{j},{ }^{H} R_{j},{ }^{V} S_{j},{ }^{V} I_{j}, t_{j}\right) \text {. }
\end{aligned}
$$

Finally

$$
\begin{aligned}
& a_{1, j, n+1}, a_{2, j, n+1}, a_{3, j, n+1}, a_{4, j, n+1}, a_{5, j, n+1} \\
& \quad= \begin{cases}n^{\alpha+1}-(n-\alpha)(n+1)^{\alpha} & \text { if } j=0, \\
(n-j+2)^{\alpha+1}+(n-j)^{\alpha+1}-2(n-j+1)^{\alpha+1}, & 1 \leqslant j \leqslant n, \\
1, & j=n+1,\end{cases}
\end{aligned}
$$

and

$$
\begin{aligned}
b_{1, j, n+1}, & b_{2, j, n+1}, b_{3, j, n+1}, b_{4, j, n+1}, b_{5, j, n+1} \\
= & \frac{h^{\alpha}}{\alpha}\left((n+1-j)^{\alpha}-(n-j)^{\alpha}\right), \quad 0 \leqslant j \leqslant n .
\end{aligned}
$$


Here, we present another numerical solution of the modified model using the numerical approximation of the Atangana-Baleanu fractional integral using the Crank-Nicolson approach.

$$
\begin{aligned}
{ }_{b}^{A B} I_{t}^{\alpha}[f(t)] & =\frac{1-\alpha}{B(\alpha)} f(t)+\frac{\alpha}{B(\alpha) \Gamma(\alpha)} \int_{b}^{t} f(j)(t-j)^{\alpha-1} d j \\
h & =\frac{T}{N}, \quad t_{l}=h l \quad(l=0,1,2,3, \cdots, N) .
\end{aligned}
$$

So

$$
\begin{aligned}
{ }_{0}^{A B} I_{t}^{\alpha}\left[f\left(t_{n+1}\right)\right]= & \frac{1-\alpha}{B(\alpha)}\left\{\frac{f\left(t_{n+1}\right)+f\left(t_{n}\right)}{2}\right\} \\
& +\frac{\alpha}{B(\alpha) \Gamma(\alpha)} \int_{0}^{t_{n+1}} f(\tau)\left(t_{n+1}-\tau\right)^{\alpha-1} d \tau \\
= & \frac{1-\alpha}{B(\alpha)}\left\{\frac{f\left(t_{n+1}\right)+f\left(t_{n}\right)}{2}\right\} \\
& +\frac{\alpha}{B(\alpha) \Gamma(\alpha)} \sum_{j=0}^{n} \int_{t_{j}}^{t_{j+1}}\left\{\frac{f\left(t_{j+1}\right)+f\left(t_{j}\right)}{2}\right\}\left(t_{n+1}-\tau\right)^{\alpha-1} d \tau \\
= & \frac{1-\alpha}{B(\alpha)}\left\{\frac{f\left(t_{n+1}\right)+f\left(t_{n}\right)}{2}\right\} \\
& +\frac{\alpha}{B(\alpha) \Gamma(\alpha)} \sum_{j=0}^{n}\left\{\frac{f\left(t_{j+1}\right)+f\left(t_{j}\right)}{2}\right\} c_{j}^{\alpha} \\
c_{j}^{\alpha}= & \frac{-\left(t_{n+1}-t_{j+1}\right)^{\alpha}+\left(t_{n+1}-t_{j}\right)^{\alpha}}{\alpha} .
\end{aligned}
$$

The numerical scheme developed above is therefore used to provide a numerical solution to the modified model in integral system form

$$
\begin{aligned}
& \frac{{ }^{\mathrm{H}} S_{\mathrm{n}+1}+{ }^{\mathrm{H}} S_{\mathrm{n}}}{2}={ }^{\mathrm{H}} S_{0}^{\mathrm{n}}(\mathrm{t})
\end{aligned}
$$

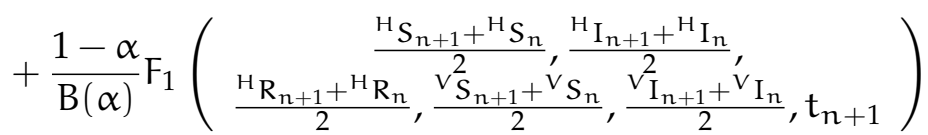

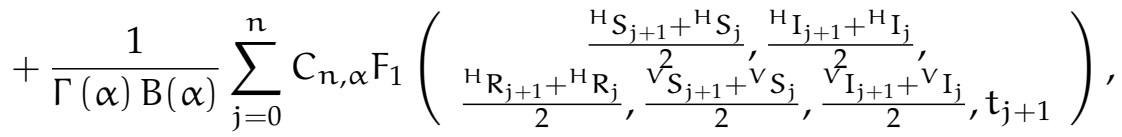

$$
\begin{aligned}
& \frac{{ }^{H} I_{n+1}+{ }^{H} I_{n}}{2}={ }^{H} I_{0}^{n}(t)
\end{aligned}
$$

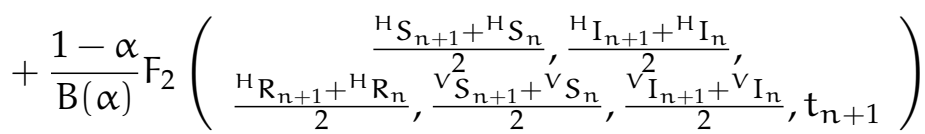

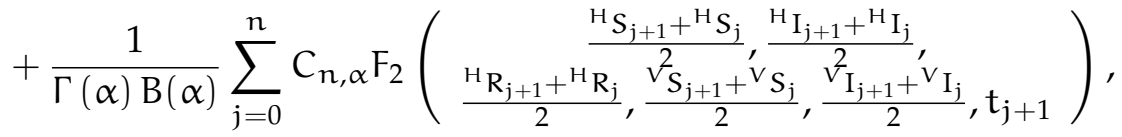

$$
\begin{aligned}
& \frac{{ }^{H} R_{n+1}+{ }^{H} R_{n}}{2}={ }^{H} R_{0}^{n}(t)
\end{aligned}
$$

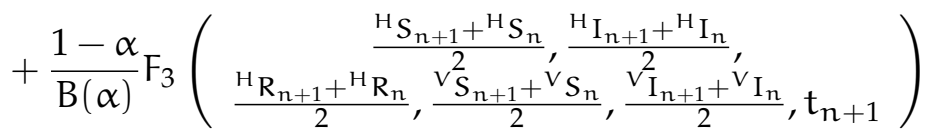




$$
\begin{aligned}
& +\frac{1}{\Gamma(\alpha) B(\alpha)} \sum_{j=0}^{n} C_{n, \alpha} F_{3}\left(\begin{array}{c}
\frac{{ }^{H} S_{j+1}+{ }^{H} S_{j}}{2}, \frac{{ }^{H} I_{j+1}+{ }^{H} I_{j}}{2}, \\
\frac{{ }_{H} R_{j+1}+{ }^{H} R_{j}}{2}, \frac{{ }^{2} S_{j+1}+{ }^{V} S_{j}}{2}, \frac{V_{I_{j+1}}+{ }^{V} I_{j}}{2}, t_{j+1}
\end{array}\right), \\
& \frac{{ }^{v} S_{n+1}+{ }^{v} S_{n}}{2}={ }^{v} S_{0}^{n}(t)
\end{aligned}
$$

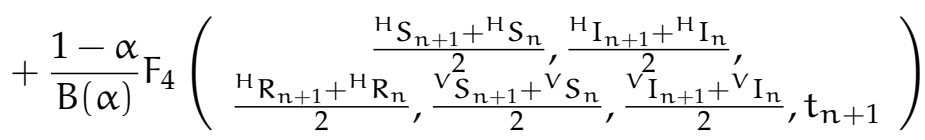

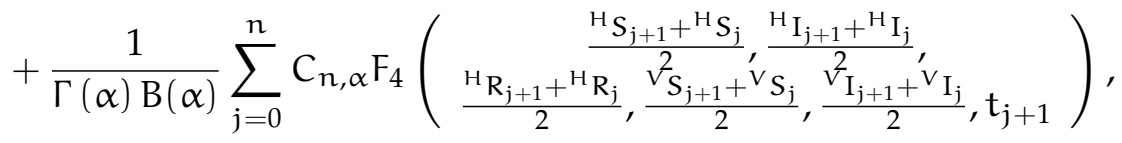

$$
\begin{aligned}
& \frac{v_{I_{n+1}}+{ }^{v} I_{n}}{2}=v_{I_{0}^{n}}(t)
\end{aligned}
$$

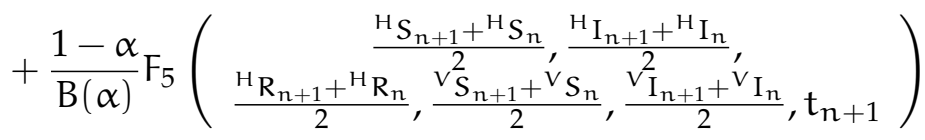

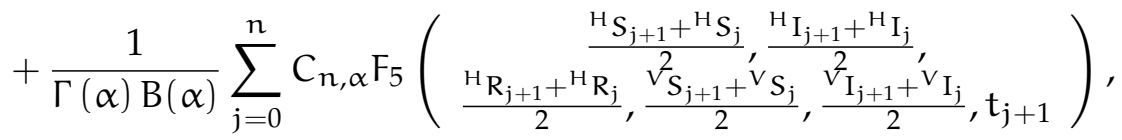

where

$$
C_{n, \alpha}=\frac{-\left(t_{n+1}-t_{j+1}\right)^{\alpha}+\left(t_{n+1}-t_{j}\right)^{\alpha}}{\alpha} .
$$

\section{Existence of solutions of system with Atangana-Baleanu sense}

In this section, we present a detailed analysis of the existence and uniqueness of the system solutions. To achieve this, we consider the following general form of fractional integral equation. Let we show the existence of solutions with linear Volterra equation.

$$
\mathrm{P}(\mathrm{t})=\mathrm{H}(\mathrm{t})+_{0}^{\mathrm{AB}} \mathrm{I}_{\mathrm{t}}^{\alpha}\{\mathrm{L}(\mathrm{t}, \tau) \mathrm{P}(\tau)\}, \quad 0<\tau \leqslant \mathrm{t} \leqslant \mathrm{T}, \quad \alpha>0 .
$$

Here Volterra equation is given by

$$
{ }_{0}^{A B} I_{t}^{\alpha}\{f(t)\}=\frac{1-\alpha}{B(\alpha)} f(t)+\frac{\alpha}{\Gamma(\alpha) B(\alpha)} \int_{0}^{t} f(y)(t-y)^{\alpha-1} d y .
$$

Also

$$
\begin{aligned}
P(t) & =\left[p_{1}, p_{2}, p_{3}, p_{4}, p_{5}\right]^{\top}, \\
H(t) & =\left[h_{1}, h_{2}, h_{3}, h_{4}, h_{5}\right]^{\top}, \\
L(t, \tau, P(\tau)) & =\left(\begin{array}{l}
L_{1}\left(t, \tau, p_{1}, p_{2}, p_{3}, p_{4}, p_{5}\right) \\
L_{2}\left(t, \tau, p_{1}, p_{2}, p_{3}, p_{4}, p_{5}\right) \\
L_{3}\left(t, \tau, p_{1}, p_{2}, p_{3}, p_{4}, p_{5}\right) \\
L_{4}\left(t, \tau, p_{1}, p_{2}, p_{3}, p_{4}, p_{5}\right) \\
L_{5}\left(t, \tau, p_{1}, p_{2}, p_{3}, p_{4}, p_{5}\right)
\end{array}\right) .
\end{aligned}
$$

We will consider

$$
\|P(t)\|=\max _{1 \leqslant i \leqslant n}\left|p_{i}(t)\right|,
$$

and

$$
L(t, s)=\max _{1 \leqslant i \leqslant n} \sum_{k=1}^{n}\left|l_{i, j}(t, s)\right|
$$


So in the proof of theorem we can use iterative formula below.

$$
\begin{aligned}
P_{n}(t) & =H(t)+_{0}^{A B} I_{t}^{\alpha}\left\{L(t, \tau) P_{n-1}(\tau)\right\}, \\
P_{0}(t) & =H(t), \\
\gamma_{n}(t) & =P_{n}(t)-P_{n-1}(t), \\
P_{n}(t) & =\sum_{i=0}^{n} \gamma_{n}(t) .
\end{aligned}
$$

Theorem 5.1. Let assume that the system given in (4.1) has a unique solution $\mathrm{P}(\mathrm{t})$ in $\mathrm{a}<\mathrm{t} \leqslant \mathrm{T}$, such that the product of kernel $\mathrm{L}(\mathrm{t}, \tau)$ and the function $\mathrm{P}(\mathrm{t})$ can be Atangana-Baleanu fractional integrable. Also inequalities are satisfied as below:

$$
\|\mathrm{H}(\mathrm{t})\|<\mathrm{h}(\mathrm{t}), \quad\|\mathrm{L}(\mathrm{t}, \mathrm{s})\|<\mathrm{l}(\mathrm{t}, \mathrm{s}),
$$

here $\mathrm{h}$ and $\mathrm{l}$ are continuous. If we have a continuous function such that

$$
\|\mathrm{P}(\mathrm{t})\|<\mathrm{p}(\mathrm{t})
$$

than following is provided

$$
p(t)=h(t)+{ }_{0}^{A B} I_{t}^{\alpha}\{L(t, \tau) f(\tau)\} .
$$

Proof. Let apply norm on both sides of (5.1).

$$
\begin{aligned}
& \|\mathrm{P}(\mathrm{t})\|=\left\|\mathrm{H}(\mathrm{t})+{ }_{0}^{\mathrm{AB}} \mathrm{I}_{\mathrm{t}}^{\alpha}\{\mathrm{L}(\mathrm{t}, \tau) \mathrm{P}(\tau)\}\right\|, \\
& \|\mathrm{P}(\mathrm{t})\| \leqslant\|\mathrm{H}(\mathrm{t})\|+\left\|_{0}^{\mathrm{AB}} \mathrm{I}_{\mathrm{t}}^{\alpha}\{\mathrm{L}(\mathrm{t}, \tau) \mathrm{P}(\tau)\}\right\|, \\
& \|\mathrm{P}(\mathrm{t})\| \leqslant\|\mathrm{H}(\mathrm{t})\|+{ }_{0}^{\mathrm{AB}} \mathrm{I}_{\mathrm{t}}^{\alpha}\|\mathrm{L}(\mathrm{t}, \tau)\|\|\mathrm{P}(\tau)\| .
\end{aligned}
$$

So we have

$$
\|P(t)\| \leqslant h(t)+{ }_{0}^{A B} I_{t}^{\alpha}\|L(t, \tau)\|\|P(\tau)\| .
$$

If we take absolute value of the exact solution and the function $p(t)$, following is obtained with conditions.

$$
p(t)-\|P(t)\|>_{0}^{A B} I_{t}^{\alpha}\|L(t, \tau)\|(p(\tau)-\|P(\tau)\|),
$$

here

$$
p(a)-\|P(a)\|>0,
$$

kernel $L(t, \tau)$ is continuous and positive for all $t \leqslant T$ then

$$
p(t)-\|P(t)\|>0,
$$

is satisfied. This completes the proof.

\section{Conclusion}

Recently, a new concept of fractional differentiation based on a non-local operator with non-singular and non-local kernel was introduced in order to better describe complex physical problems that follows at the same time the power and exponential decay law. The concept has been used to model some physical problem arising in the field of non-linear dynamic, thermal science, mechanical engineering, etc. This concept is proven to be powerful mathematical tool to model many physical phenomena better than the existing concept of fractional differentiation based on the power decay law. Very recently, the world witness a strange spray of a transmissible fatal disease known as Zika virus, in particular in Brazil, United State of America and some fewer countries in Europe and Africa. Due to the fatal effect of this virus, some athletes were frozen their reproductive cell in case they were infected during the Olympic Games Brazil 
2016. Many theoretical studies based on mathematical concepts have not been done intensively for this dynamical system. In this work, we have considered the model of Zika spread using the concept of fractional differentiation with non-local and non-singular kernel to include into mathematical formulation the complexity of the spread. The existence and uniqueness of a system solutions under some conditions were established. The model was solved numerically using the well-known Adams type predictor-corrector rule for Atangana-Baleanu fractional integral. Some numerical simulations are presented.

\section{Acknowledgment}

The authors extend their sincere appreciations to the Deanship of Science Research at King Saud University for funding this prolific research group PRG-1437-35.

\section{References}

[1] O. J. J. Algahtani, Comparing the Atangana-Baleanu and Caputo-Fabrizio derivative with fractional order: Allen Cahn model, Chaos Solitons Fractals, 89 (2016), 552-559. 2

[2] B. S. T. Alkahtani, Chua's circuit model with Atangana-Baleanu derivative with fractional order, Chaos Solitons Fractals, 89 (2016), 547-551. 1

[3] A. Atangana, D. Baleanu, New fractional derivatives with non-local and non-singular kernel: theory and application to heat transfer model, Therm. Sci., 20 (2016), 763-769. 2

[4] A. Atangana, D. Baleanu, Caputo-Fabrizio derivative applied to groundwater flow within confined aquifer, J. Eng. Mech., 143 (2017), D4016005-01-D4016005-05. 2

[5] A. Atangana, I. Koca, Chaos in a simple nonlinear system with Atangana-Baleanu derivatives with fractional order, Chaos Solitons Fractals, 89 (2016), 447-454. 1

[6] E. Bonyah, K. O. Okosun, Mathematical modeling of Zika virus, Asian Pac. J. Trop. Dis., 6 (2016), 673-679. 1, 3

[7] L.-P. Chen, J.-F. Qu, Y. Chai, R.-C. Wu, G.-Y. Qi, Synchronization of a class of fractional-order chaotic neural networks, Entropy, 15 (2013), 3265-3276. 1

[8] J. Hadamard, Essai sur l'étude des fonctions données par leur développement de Taylor, J. Mat. Pure Appl. Ser., 4 (1892), 101-186. 1

[9] U. N. Katugampola, New approach to a generalized fractional integral, Appl. Math. Comput., 218 (2011), 860-865. 1

[10] J. Lessler, L. H. Chaisson, L. M. Kucirka, Q.-F. Bi, K. Grantz, H. Salje, A. C. Carcelen, C. T. Ott, J. S. Sheffield, N. M. Ferguson, D. A. Cummings, Assessing the global threat from Zika virus, Science, 353 (2016), 663-673. 1

[11] F. Mainardi, Y. Luchko, G. Pagnini, The fundamental solution of the space-time fractional diffusion equation, Fract. Calc. Appl. Anal., 4 (2001), 153-192. 1

[12] O. O. Makinde, K. O. Okosun, Impact of chemo-therapy on optimal control of malaria disease with infected immigrants, Biosyst., 104 (2011), 32-41. 1, 3.1

[13] O. O. Makinde, K. O. Okosun, Modelling the impact of drug resistance in malaria transmission and its optimal control analysis, Int. J. Phys. Sci., 6 (2011), 6479-6487. 1, 3.1 